\title{
Design and Evaluation of the Probation/Parole and Reentry Coach App (PARCA): A Platform to Support a Successful Reentry into Community
}

\author{
Lidan Zhang \\ Worcester Polytechnic Institute \\ lzhang11@wpi.edu \\ Nick Richardson \\ RTI International \\ nrichardson@rti.org
}

\author{
Bengisu Tulu \\ Worcester Polytechnic Institute \\ bengisu@wpi.edu \\ Steve Shoptaw \\ University of California, Los \\ Angeles \\ sshoptaw@mednet.ucla.edu
}

\author{
Steven Jenkins \\ Q2i \\ sjenkins@,q2i-group.com \\ Faye Taxman \\ George Mason University \\ ftaxman@gmu.edu
}

\begin{abstract}
Justice-involved people (JIP), especially those with substance use disorder (SUD), face multiple challenges and inadequate resources as they re-enter their communities post-incarceration. Technology interventions have proven to be feasible in supporting this unique population. In this study, we applied iterative development methodology and user-centered design to develop and evaluate a technology artifact called Probation/Parole and Reentry Coach App (PARCA) composed of a JIP mobile app and justice team (JT) portal. PARCA assists recently released JIP with SUD with their reentry. We completed three feedback cycles involving healthcare and criminal justice experts and JIP $(N=16)$ in design workshops, interviews, and usability studies. We collected and analyzed qualitative (semi-structured interviews) and quantitative (System Usability Scale and app use) data. As a pilot study that focuses on qualitative observations, the results indicate that PARCA provides an excellent usability experience for JIP (SUS scores $>80$ ) and is useful and satisfactory (based on qualitative responses).
\end{abstract}

\section{Introduction}

With over 10 million arrests[1] and around 2.3 million people in the United States (U.S.) incarcerated [2], justice-involved people (JIP) represent a large subpopulation. JIP recently released from incarceration normally face a challenging environment and numerous barriers that prevent successfully entering society. Up to $85 \%$ of the prison population has either an active substance use disorder (SUD) or was incarcerated for a crime involving drugs or drug use [3]. When combined with common challenges faced by JIP, such as housing stability and employment challenges [4], SUD can significantly affect the outcomes of the reentry process [5].

JIP receive few preparation opportunities during incarceration and lack access to localized resources to assist their reentry, which can facilitate substance abuse, crime, and recidivism [6]. From 1999 to 2009, 14.8\% of former prisoner deaths were related to opioids [7]. About $50 \%$ of JIP post-incarceration are likely to be arrested within the first year, $68 \%$ within three years, and $77 \%$ within five years after their release [8]. Interventions that facilitate JIP's access to SUD treatment, support services, and other resources have the potential to address access issues or inequitable service delivery.

Diverse delivery mechanisms, such as face-to-face, phone calls, text messaging, emails, or mobile apps, that support JIP's reentry are available. Despite their effectiveness, face-to-face in-person interventions can be more costly [9] and less accessible [10] compared to technology interventions. The interventions using phone calls are prevailing and found to be effective [11]. However, they require both intervention providers and JIP to be available simultaneously. Many JIP have unpredictable schedules and unstable housing. Hence, the synchronous communication requirement introduces a barrier for interventions delivered by phone calls.

The interventions using smartphones, e.g., text messaging and apps $[12,13]$, allow JIP to interact with service providers at anytime from anywhere. Hence, they potentially can scale better and assist more JIP than in-person methods. Mobile phone ownership reached $97 \%$ and smartphone ownership went from $35 \%$ in 2011 to $85 \%$ in 2021 in the U.S. [14]. Most JIP have access to smartphones despite the variations across socioeconomic groups [15]. The rapid increase in smartphone ownership brings new opportunities to deliver interventions that address multiple issues JIP 
face [16]. Prior mobile technology intervention studies for JIP highlighted easy accessibility and effectiveness in their findings.

Although we identified various technology interventions in the literature, most studies targeted a single issue in isolation. Some focused on SUD treatment and others focused on mental health disorders $[12,17]$. A few studies targeted helping JIP with job search, technical literacy, and case management [1820]. To the best of our knowledge, no study focused on housing, financial support, childcare, food, and transportation. Although these interventions proved to be useful in improving JIP's reentry, this monotonous focus may not be sufficient in identifying the most effective digital interventions for JIP.

The study aims to design and evaluate a holistic and centralized solution artifact that attempts to address all the aforementioned factors simultaneously to improve community reentry. We followed user-centered design [21] and iterative development methodology to develop our artifact. Our solution is an all-in-one platform that (a) provides localized resources about SUD treatment, healthcare services, job search, housing, financial support, childcare, food, and transportation, (b) enables continuous case management, and (c) facilitates treatment engagement through useful features such as calendar and journaling.

\section{Literature Background}

\subsection{The Challenges and Barriers JIP Face}

Social disparities JIP face dramatically elevates behavioral health disorders in this population [22]. Around $56 \%$ of JIP have past-year mental health problems [23], 66\% have substance use disorders [24], $13-20 \%$ have lifetime suicide attempts [25], 15\% have lifetime opioid use, and $11 \%$ have pain medication dependence [26].

Given that JIP have 4 to 9 times the rate of SUD than the general population and 4 times the rate of mental health disorder, substance abuse has been criminalized, and the number of JIP with SUD has been increasing [27]. Moreover, mental health and substance use disorders (SUD) are associated with overdose [28], suicide [25], disabilities, and physical disorders [29], homelessness [30], repeat incarcerations [31], and death [32]. Research shows a high likelihood of drug-related death immediately after release, especially during the first few months [33]. The increasing number of JIP with SUD emphasizes the urgency to access treatment and provides opportunities to intervene in JIP who would rather not seek treatment [34].

The justice system is ill-equipped to handle behavioral health disorders given the lack of adequate programs and services available to those that are justiceinvolved. Lack of access to treatment and services during incarceration and post-incarceration results in common occurrences of relapse and premature death [35]. Although jails are the largest mental health service providers, treatment availability is still scarce [36].

The needs of JIP with SUD are complex and vary considerably across individuals. Multiple studies suggested that SUD treatment is effective in reducing substance use and recidivism [37], but most JIP with SUD do not receive treatment during or after incarceration. Only $28 \%$ of JIP in prison and $22 \%$ of JIP in jail with drug use disorders participated in treatment while incarcerated [38], and less than $50 \%$ of JIP with SUD continuously received SUD treatment one year after release [39]. The low participation in SUD treatment may impact the need or desire to engage in other types of services. Less than $8 \%$ of JIP can access community-based services [40]. Even for those JIP that do have access to the treatment, the coordination of care and available programs is hard to achieve because some jurisdictions are often lacking significant resources [41].

JIP's criminal convictions and poor health conditions limit their employment prospects, public housing assistance, and social services [42]. When they are released from incarceration, most of them immediately face challenges of employment, healthcare, and housing. These issues are normally bundled together. For instance, intense health issues and consequent behavioral problems of JIP are the major causes of homelessness [43]. In turn, unstable housing can decrease the likelihood of remaining in substance abuse treatment [44]. Research warns that homelessness exacerbates existing health issues and creates new issues, and recovery becomes more difficult without housing [43]. Failure to address these reentry challenges might put JIP at risk of rearrest and recidivism [45]. Addressing all the social determinants of health (SDOH) factors simultaneously could minimize the barriers and ensure efficient outcomes.

It is essential for JIP to navigate through various community resources, e.g., SUD treatments, employment, housing, financial support, childcare, food, and transportation. If newly released JIP with SUD do not receive adequate assistance and resources, they have no choice but to use the limited monetary assistance to fulfill basic needs such as food, transportation, and housing first [46], rather than receive treatments. Sometimes, JIP have to sacrifice treatments. However, some treatments, especially SUD treatments, are big needs for this population [47]. Hence, equipping JIP with SUD, especially those recently released, with necessary services and support tools is important. The assistance and support tools could be the key to address their underlying problems, ensure treatment retention, 
and can facilitate JIP to successfully reenter into their communities.

\subsection{Technology-Based Interventions for JIP}

Digital health technology can help recently released JIP navigate their new environment and adapt to the changes that occurred during their incarceration. Technology-based interventions can be tailored to specific needs. Many commercially available health programs (e.g., Fitbit, MyFitnessPal, Weight Watchers) already use such strategies to provide feedback, goal setting, reminders, and facilitation of social connections. The Motivational Assessment Program to Initiate Treatment (MAPIT) clients who set goals and reminders had greater rates of early treatment initiation and reduced substance use compared to clients who did not [48]. Moreover, digital technology systems also improve the quality of provider interactions. Many studies demonstrate that provider-initiated systems can help improve the quality of healthcare interactions [49] and encourage clients to discuss intervention material with their providers [13].

Scientific evidence indicates that technology interventions can be as effective and beneficial as inperson delivered interventions. One study showed that an internet-based cognitive-behavioral intervention for depression is equally beneficial to regular face-to-face therapy [50]. Another study noted that brief alcohol screening and intervention for college students intervention can be effectively delivered via telehealth compared to face-to-face brief alcohol interventions [51]. Technology interventions utilizing phone calls and text messages were available in the literature. For example, one study supported that setting short-term goals and receiving text or email reminders about their probation and treatment goals increase the likelihood of SUD treatment initiation [12].

Nevertheless, the technology interventions using synchronous interactions may have limited impact due to missed opportunities caused by conflicting commitments. Interventions using mobile apps or websites can be designed to limit synchronous interactions, and hence have the potential to become another option that could assist more JIP.

Substantial literature is available on the effectiveness of asynchronous technology interventions at reducing substance use and related risky behaviors [52]. In the MAPIT study, a web-based intervention was found to facilitate increased motivation for SUD treatment and HIV care through combining web-based sessions about motivation, goal setting, coping strategies, and social support, with emails or text reminders [13]. StaySafe, a self-administered tabletbased app, was found to be feasible and have high levels of satisfaction for teaching better decision-making skills regarding health risk behaviors [53]. A case management smartphone app, Link2Care, is currently being investigated to improve connections with case managers to increase treatment utilization and reduce homelessness [18]. Technology makes it easier to provide localized support, such as access to locationspecific information about risk reduction, housing, accessing medical care, SUD treatment program options and availability, as well as support groups for JIP postincarceration.

\section{Methodology}

This project was designed as a pilot qualitative study, where the goal was to create a proof-of-concept product. We followed user-centered design [27] and iterative development methodology to develop a holistic mHealth platform - Probation/Parole and Reentry Coach App (PARCA).

\subsection{Iterative Development Methodology}

The study was approved by the Institutional Review Board (IRB) of Worcester Polytechnic Institute (WPI). We conducted 3 feedback cycles (Figure 1) to develop and evaluate the platform prototypes and collect feedback from 10-20 key informants to identify the majority of the usability issues through system usability scale (SUS) [54] scores and qualitative interviews.

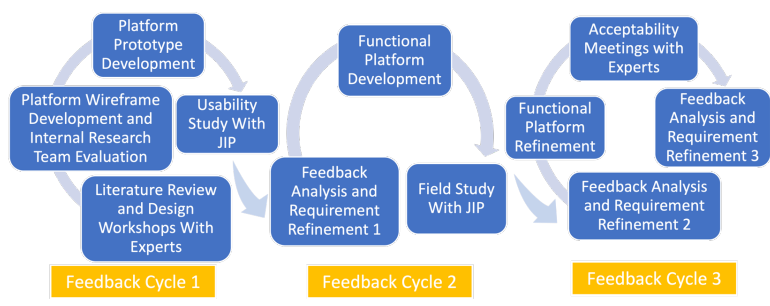

Figure 1. Iterative development methodology design flow

Participants were included in the usability study if they were (1) justice-involved adults with SUD, (2) released from incarceration within 3 months, (3) with access to iPhone or Android smartphones, and (4) living in Nashua, NH, or Worcester, MA at the time of the study. Participants were excluded from the study if they were (1) medically/ cognitively unable to participate in screening or assessment (e.g., psychosis, hostile behavior, severe pain), (2) already enrolled during the previous visit, or (3) no access to a reliable telephone for follow-up. 
We created semi-structured interview protocols and use scenarios to be followed by the participants in all usability studies. In the first feedback cycle, we developed wireframes and a low fidelity platform prototype and assessed it in three focus groups. Each focus group lasted about an hour. First focus group participants $(\mathrm{N}=4)$ were recruited from Worcester, MA. Second and third focus group participants $(\mathrm{N}=3$ and $\mathrm{N}=2$ respectively) were recruited from Nashua, $\mathrm{NH}$. In the second feedback cycle, we developed the functional platform and conducted internal user acceptance testing followed by a field usability study with seven participants recruited from Nashua, NH. In the third feedback cycle, we refined the functional platform and held stakeholder acceptability meetings with the justice team and healthcare experts in the field. Table 1 presents the demographics of the participants involved in the focus groups and the field study.

Table 1. Participant characteristics

\begin{tabular}{llll}
\hline Study & Gender & Race & Ethnicity \\
\hline Usability & 9 Males & 3 Black & 4 Hispanic \\
Study & & 6 White & 5 Unknown \\
\hline Field & 6 Males & 4 Black & 2 Hispanic \\
Study & 1 Female & 3 White & 5 non- \\
& & & Hispanic \\
\hline
\end{tabular}

\section{Results}

We created an innovative mHealth platform as the artifact to improve JIP's reentry into communities and increase access to SUD treatment by providing features that facilitate goal setting, progress tracking, access to resources, communication opportunities, journaling, and notifications. This artifact named PARCA is composed of 2 sub-systems: a client-facing mobile app for JIP, and a dashboard portal for the justice team (JT). This artifact was developed using self-determination theory [55] to support JIP with SUD as they reenter into their communities.

\subsection{Feedback Cycle 1: Initial Platform Wireframes and Platform Prototype Design}

4.1.1. The Design of the Initial Wireframes. We conducted a literature review of the criminal justice domain, substance use disorder domain, and behavior change domain. We held design workshops with experts to identify JIP's needs and the initial design requirements. We started with the members of the research team who are experts in criminal justice, substance use disorder treatment, and health information technology development. Next, we reached out to experts who hold prominent positions in various justice and health and human services departments including, VT, MA, CA, WV, CO, and AZ, supporting JIP post-incarceration. We also met with stakeholders, including case managers and case coordinators from the judicial system, community health center, community housing, employment support services, and recently released individuals with SUD. We learned how JT works with JIP and what they want from the platform. The JT was interested in the calendar feature, the telehealth feature, especially for mental health, and the feature to track treatment adherence. They wanted the app to allow them to upload JIP's case plans. They also wanted to have an additional way to communicate with JIP and have access to service providers.

After analyzing the meeting notes from various workshops, we created the initial requirements and developed the initial platform wireframes. Not all the requirements were included in the prototype (e.g., access to service providers). We designed the initial platform wireframes to include a JT portal and a client mobile app for JIP. Our initial design requirements for the JT portal included providing access to client-level data about selected goals and progress towards these goals in the form of small steps and allowing the JT to share resources, case management plans, appointment schedules with JIP. After receiving positive feedback from JT, we kept the original JT portal design and made only minor style changes to it during the app prototype iterations.

Our initial design requirements for the mobile app includes (1) a risk assessment questionnaire which is useful in justice settings, (2) resource information related to SUD treatment, housing, transportation, child care, food pantries, and financial planning, and employment, (3) appointment scheduling and tracking, (4) profile creation, e.g. username and passwords creating, case plans checking, etc., (5) options of setting top 3 goals with small steps (6) progress tracking regarding clients' goals and steps, and (7) asking additional resources from JT. The justice system uses the actuarial risk assessment questionnaire to prioritize care for individuals. The resources were provided in different features. In the goal-setting feature, relevant resources were listed under each step by listing names of relevant organizations' or services', their addresses that can be viewed in Google maps with a click, and contact numbers with click-to-call function. Users could also find more categorized resources under the "Resources" feature, such as housing, SUD treatment, employment, food, transportation, child care, and financial services. Each resource provides related service information as described above. If users want additional resources, they can go to the "Message" feature and click any categorized resources to send a resource request to a member of the JT. Once the JT 
receives resource requests, JT replies in a response message with the additional resource information. Figure 2 illustrates the initial platform wireframes that address these requirements and use scenarios.

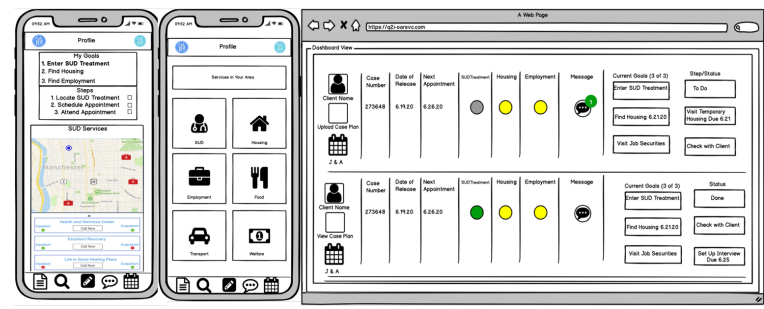

Figure 2. Initial platform wireframe examples and JT portal 1

4.1.2. The Design of the Platform Prototype. We presented the platform wireframes and collected opinions from the research team. After communicating with experts and collecting the feedback about the platform wireframes in Figure 2, we created the first platform prototype presented in Figure 3. In the new prototype, we updated some design requirements for the mobile app including (i) allowing clients to create journaling, (ii) providing an option of sharing data or not, and (iii) providing "healthcare services" as a new resource category. We also added journaling in the JT portal for JT to have access to client-level data if the clients choose to make this information available. Moreover, based on the MAPIT intervention, we included language that was motivating and focused on helping JIPs examine their choices. The language reflects motivational efforts to address ambivalence about seeking care.

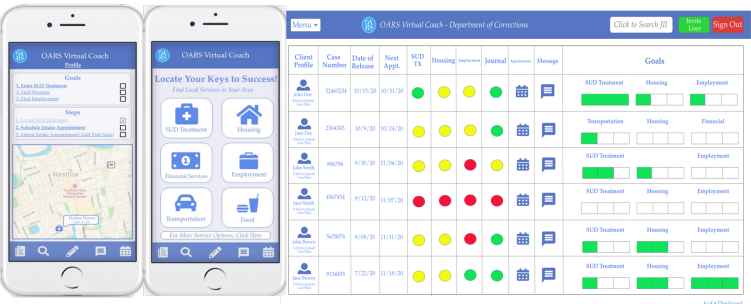

\section{Figure 3. Platform prototype examples and JT portal 2}

4.1.3. The Usability Study with JIP. We started the focus groups by asking participants about their incarceration journey and technology experience. Next, we asked them to review the mobile app prototype and complete tasks based on the use scenarios and we observed their interactions with the app prototype. At the end of each focus group, we asked questions about the app's usefulness, ease of use, usability based on their experience during the focus group.

\subsection{Feedback Cycle 2: Functional Platform Design}

4.2.1. Usability Study Feedback Analysis and Requirement Refinement. The research team analyzed feedback from the usability study to identify issues that need to be addressed and potential new features that could be added to the requirements. The participants liked ranking and setting up goals with relevant resources, the reminders and notifications, the daily log feature, the easy-to-understand language, design structures in the app, and goal recommendations provided by the app. They noted willingness to share information with their reentry coordinator and other trusted people. In terms of use frequency, some anticipated checking the mobile app daily and others indicated that they would check based on their needs. During the second and third focus groups, a strong opposition to the risk assessment questionnaire was observed. The participants in the second focus group perceived the questions as offensive and unrelated, and they indicated that they would prefer to answer questions that corresponded with the goal categories. The participants of the third focus group chose to skip the risk assessment questionnaire completely.

Participants made a number of suggestions including (1) having motivational messages or videos in the mobile app, (2) making the app more accessible for illiterate people, e.g., adding an audio function (3) setting the app bilingual, e.g., adding Spanish as a language option, (4) adding personal, social and local events in the calendar (e.g., doctor appointments, baseball games), (5) adding "Education" as a category in the resource and including occupational training opportunities, (6) expanding the top 3 goals to 5 goals, (7) providing additional feedback regarding the journals they created in the daily log feature, (8) providing specific suggestions, creating a task list to fix unmet goals, or allowing edits to the unmet goals, and (9) having options to control information sharing preferences.

After reviewing the feedback, the research team decided to refine the prototype by (i) adding pop-up motivational quotes in the app, (ii) removing the risk assessment questionnaire, (iii) providing general recommended goals (SUD treatment, employment, and housing), and (iv) adding "Education" in the resource categories. All the changes were implemented in the functional platform development.

4.2.2. The Design of the Functional Platform Prototype. We implemented new changes in the functional platform prototype based on new requirements and conducted internal user acceptance testing with three beta users to identify UI issues, bugs, 
content errors. Using the results of the internal testing, we made some improvements in the platform functionality. For example, instead of asking users to rank goals from 1 (the most important) to 7 (the least important), we decided to select the top 3 goals to minimize clicks. Table 2 shows the platform features developed during this study. The functional platform prototype and the system architecture of this platform are illustrated in Figure 4 and Figure 5 respectively.

Table 2. System components and platform Features

\begin{tabular}{|c|c|c|}
\hline $\begin{array}{l}\text { System } \\
\text { Components }\end{array}$ & $\begin{array}{l}\text { Portal } \\
\text { allows the } \\
\text { JT to: }\end{array}$ & $\begin{array}{l}\text { Mobile App } \\
\text { allows JIP to: }\end{array}$ \\
\hline 1. Goal setting & $\begin{array}{l}\text { View and } \\
\text { track JIP } \\
\text { selected goals }\end{array}$ & $\begin{array}{l}\text { Set three goals to } \\
\text { address in the next } \\
\text { month }\end{array}$ \\
\hline $\begin{array}{l}\text { 2. Progress } \\
\text { tracking }\end{array}$ & $\begin{array}{l}\text { View and } \\
\text { track steps to } \\
\text { accomplish } \\
\text { goals }\end{array}$ & $\begin{array}{l}\text { Track progress } \\
\text { towards goals by } \\
\text { completing subtasks } \\
\text { necessary to } \\
\text { accomplish a goal }\end{array}$ \\
\hline $\begin{array}{l}\text { 3. Profile } \\
\text { managing }\end{array}$ & $\begin{array}{l}\text { Upload and } \\
\text { adjust JIP } \\
\text { case plan }\end{array}$ & $\begin{array}{l}\text { Access location- } \\
\text { specific information } \\
\text { directly related to } \\
\text { goals (website, } \\
\text { phone number, } \\
\text { address) }\end{array}$ \\
\hline $\begin{array}{l}\text { 4. Access to } \\
\text { resources }\end{array}$ & $\begin{array}{l}\text { Provide } \\
\text { information } \\
\text { on support } \\
\text { services to the } \\
\text { JIP }\end{array}$ & $\begin{array}{l}\text { Request additional } \\
\text { resources from JT }\end{array}$ \\
\hline $\begin{array}{l}\text { 5. Journaling } \\
\text { and } \\
6 . \text { Notifications }\end{array}$ & $\begin{array}{l}\text { Manage and } \\
\text { schedule } \\
\text { appointments } \\
\text { for JIP }\end{array}$ & $\begin{array}{l}\text { Keep daily logs, } \\
\text { receive secure } \\
\text { messages }\end{array}$ \\
\hline $\begin{array}{l}7 . \\
\text { Communication } \\
\text { opportunities }\end{array}$ & $\begin{array}{l}\text { Send secure } \\
\text { messages to } \\
\text { JIP }\end{array}$ & $\begin{array}{l}\text { Access case plan } \\
\text { and scheduled } \\
\text { appointments }\end{array}$ \\
\hline
\end{tabular}

4.2.3. The Field Study with JIP. At the end of feedback cycle 2 , we conducted a 2-week field usability study to evaluate the functional platform and refine the requirements. We set up anonymous test accounts for participants to $\log$ in during interviews. We asked participants about their reentry journey, and the challenges they are facing. Next, we assisted them to install the app, walked them through the app prototype, and asked them to finish 3-5 use scenario tasks covering key functionalities independently using the test accounts. At the end of the session, the participants were guided in creating their own accounts to use for the 2week usability study. We asked for their feedback regarding the app's features, usefulness, and usability assessed by qualitative questions and quantitatively with the SUS, an independent and powerful tool to assess the usability of the app. At the end of the session, participants were asked to use the app on their smartphones for 2 weeks. We collected app use data during the 2-week period and conducted exit phone interviews after the 2 -week period ended to get more qualitative feedback about their experience with the app.

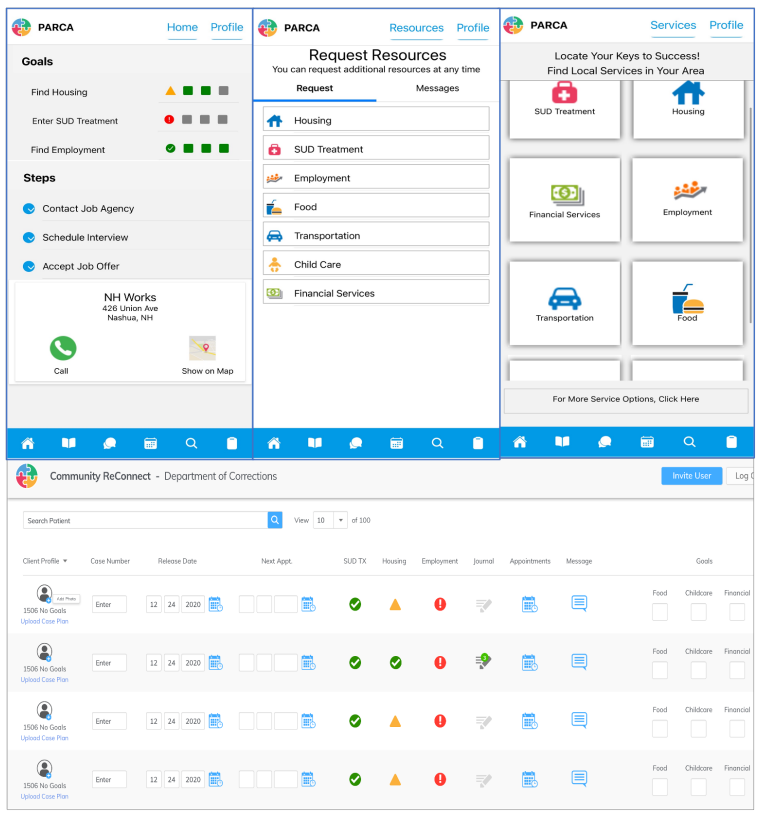

Figure 4. Functional platform prototype examples and JT portal 3

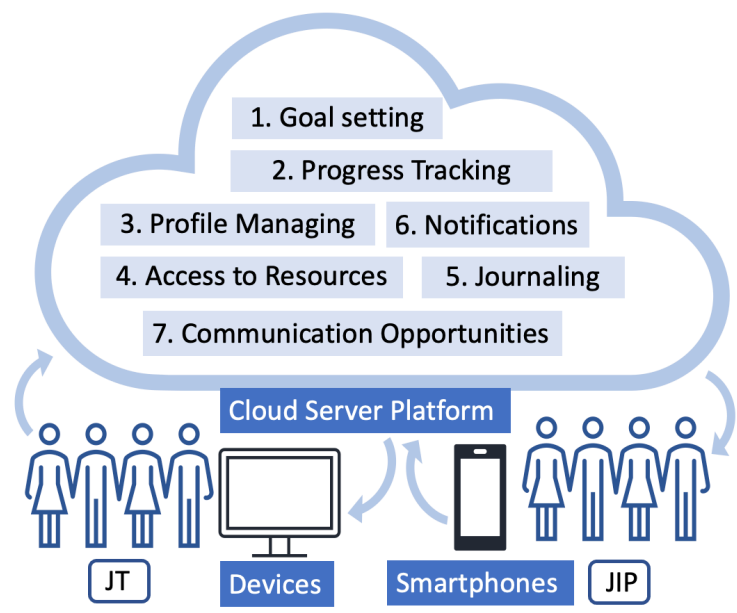

Figure 5. The system architecture of the artifact

\subsection{Feedback Cycle 3: Functional Platform Refinement and Results Communication}

4.3.1. Field Study Feedback Analysis and Platform Refinement. Analysis of the qualitative feedback from the initial interviews during the field study revealed that all participants were experienced with smartphones, but 
only one participant had computer experience. Participants had a positive attitude about the app and stated that the app was easy to use and the features provided satisfied their expectations. The participants highlighted that the goal-setting feature was beneficial for them to remember and make progress towards their goals. Requesting new resources, viewing their appointments and case plan, and receiving notifications about upcoming appointments directly through the app were features that the participants found useful and important. Most of the participants indicated that they were willing to share goals and progress with their case managers. They noted that they would check the app based on their needs. The participants were mainly concerned about basic needs, such as housing, financial services, and jobs. Many of them also indicated that they preferred communicating with real people when we asked them if they would like to have an automated chatbot responding to their resource requests.

Three participants had difficulty with creating accounts and logging in, and two participants were confused about the "SUD treatment" and "Financial services" terms. The participants made various suggestions including (1) adding a 24-hour helpline for minor emergencies, (2) having a counselor for SUD treatment available through messaging, (3) tightly integrating resources within the app, e.g., ability to conduct job search or apply for jobs, and (4) providing more resources and services in the home page.

In addition to the qualitative feedback, we collected responses to the SUS questionnaire [54] at the end of the initial interviews. The platform received an average SUS score of 97 out of 100, which indicates high acceptability and excellent usability. This indicator presents that the app is satisfactory, functionally usable, and can fit into the context of reentry management.

A total of four participants out of seven finished the exit interviews. We added a new question about stigma in the exit interview proposal, and all of them indicated that having a feature that allows them to log their stigma experiences is a good idea. Some participants mentioned that the daily log feature was helpful for them to reflect on their day and to take notes on their progress. The convenience of having access to appointments and reminders was also mentioned as a strength of the app. After two weeks of use, one participant mentioned that the progress report was not available in the app, although it was presented in the home page. In addition, two participants thought that the resources provided in the app were not sufficient and could be enhanced. Participants suggested adding medical refills and women assistance.

App-use data was derived from system logs created through tracking login activity, journals created, services requested, steps completed, and goals completed. During 98 study days across 7 participants, 51 unique logins occurred. The accumulative interaction with the app in the 14 days ranged from 1 to 29 . Two participants created 20 journal entries, 3 participants made 5 service requests and 2 participants completed a total of 4 steps during the 2-weeks use period, and 2 participants did not return to the application.

After analyzing the qualitative data from the interviews, the research team discovered that employed JIP might have difficulty attending a job interview or meeting with employment support specialists during working hours. This might limit JIP's ability to seek better employment opportunities, hence providing features that enable JIP to conduct job search and see support through the app could be beneficial in future iterations. We also noted that some participants lacked motivation to use the app during the 2 -week field study.

4.3.2. Result Communication and Feedback Collection. We conducted meetings with potential stakeholders and JT experts from healthcare, community services, and justice teams representing seven states in the U.S. (CA, WV, MA, VT, PA, TX, and $\mathrm{AZ}$ ). During these meetings, we presented our preliminary findings and asked their feedback about the acceptability of the platform, opportunities the platform could facilitate, and challenges and barriers that the platform could address in their context. The collective feedback indicates that the platform is useful, usable, comprehensive, and could potentially be effective. The platform features, such as scheduling appointments and messaging directly through the platform provide value for all stakeholders. Technologies like this one are needed, especially during the COVID19 pandemic. Some of the JTs were already implementing digital apps. However, they noted concerns around the cost of implementation and limited budgets for innovation, loss of confidentiality of the JIP information in 3rd party app platforms, the difficulty of keeping in touch with JIP after release and engaging them in treatment, and challenges in collaboration between justice team members spread across different departments in the organization.

\section{Discussion and Conclusion}

JIP, especially those with SUD, face multiple challenges that are barriers to being successful in the community after a period of incarceration. While receiving few preparation opportunities, they lack access to localized services to assist their reentry. To improve JIP's reentry into communities, we have developed a mHealth platform, including a client mobile app and a justice team portal, as an efficient holistic digital solution. We utilized user-centered design 
approach [21] and iterative development methodology to design and develop the artifact. We created user requirements, designed platform wireframes, and developed platform prototypes. We evaluated platform prototypes for usability and acceptability using focus groups with JIP and interviews with other stakeholders. The functional platform, providing features such as goal setting, process tracking, profile managing, resource accessibility, journal creating, notification, and communication, is available on the app stores and is ready for effectiveness testing.

Although a feasibility trial has not yet been conducted, the initial deployment of the app yielded strong qualitative and quantitative data from participants describing the app as usable, useful, and satisfactory. The mobile app has the potential to improve JIP's self-management, SUD treatment engagement, and service accessibility. This approach has the potential to empower JIP to increase personal responsibility and accountability for their behavior during reentry, which has been shown to have positive outcomes. Meanwhile, the JT portal can facilitate asynchronous communication between the justice team and the JIP through messaging functionality. The JT portal provides additional channels for JT to track JIP's reentry progress and provide assistance. The JT portal dashboard visualizes areas where additional help is needed and areas where progress is being made. Through increased connection, vision, and support the platform aims to help justice teams and individuals improve community integration. This study team developed a holistic artifact utilizing system analysis and platform design, which could be a potential advanced step for integrating technology with JIP reentry programs.

This study was conducted during COVID-19 lock downs in 2020-21, which forced us to modify our original study protocol and limit in-person interactions we could conduct with the participants trialing prototype versions of the app. Despite these limitations, we were able to observe users' interactions with app prototypes during virtual meetings. At the end of feedback cycle 2 , we collected SUS, the only quantitative measure in the study. We were able to achieve consistently high SUS scores from the first 7 participants (average SUS=97) after they experienced the app prototype independently. Literature indicates that $80 \%$ of usability problems can be identified with as few as 10 subjects [56], hence the quantitative and qualitative data collected in this study indicates that the usability experience provided by the mobile app is acceptable.

In future studies, we plan to expand the implementation of the app to a larger sample size and longer study period to measure the effectiveness and acceptability of the app. We will also test and empirically measure the efficacy of the app in improving SUD treatment access, initiation, and retention through better data, streamlining workflows, coordination of services, and developing more robust local partnerships that are needed to address SDOH issues and improve population health.

\section{Acknowledgements}

Research reported in this publication was supported by the National Institute on Drug Abuse of the National Institutes of Health under Award Number R41DA051156. The content is solely the responsibility of the authors and does not necessarily represent the official views of the National Institutes of Health.

\section{References}

[1] Federal Bureau of Investigation, "Persons Arrested", in 2018. [Online]. Available: https://ucr.fbi.gov/crime-inthe-u.s/2017/crime-in-the-u.s.-2017/topicpages/persons-arrested. [Accessed 08/19, 2021]

[2] W. Sawyer and P. Wagner, "Mass Incarceration: The Whole Pie 2020 ", in 2020. [Online]. Available: https://www.prisonpolicy.org/reports/pie2020.html. [Accessed 2/15, 2021]

[3] National Institute on Drug Abuse, "Criminal Justice DrugFacts", in 2020. [Online]. Available: https://www.drugabuse.gov/publications/drugfacts/crimi nal-justice [Accessed 2/15, 2021]

[4] L. Lovoy, "Life After Prison: Ex-Felons Often Struggle to Find a Job", in 2014. [Online]. Available: https://wbhm.org/feature/2014/life-after-prison-exfelons-often-struggle-to-find-a-job/. [Accessed 2/15, 2021]

[5] H. J. Holzer, S. Raphael and M. A. Stoll, "Employment barriers facing ex-offenders", Urban Institute Reentry Roundtable, 2003, pp. 1-23.

[6] C. A. Visher and K. Mallik-Kane, "Reentry experiences of men with health problems", Springer, 2007, pp. 434-460.

[7] I. A. Binswanger, P. J. Blatchford, S. R. Mueller and M. F. Stern, "Mortality after prison release: opioid overdose and other causes of death, risk factors, and time trends from 1999 to 2009", Annals of internal medicine, 2013, pp. 592-600.

[8] P. A. Langan and D. J. Levin, "Recidivism Of Prisoners Released In 1994", in 2002. [Online]. Available: https://www.bjs.gov/index.cfm?ty=pbdetail\&iid=1134. [Accessed 4/3, 2021]

[9] A. J. Cowell, G. A. Zarkin, B. J. Wedehase, J. Lerch, S. T. Walters and F. S. Taxman, "Cost and cost-effectiveness of computerized vs. in-person motivational interventions in the criminal justice system", Journal of substance abuse treatment, 2018, pp. 42-49.

[10] J. Unützer, W. Katon, M. Sullivan and J. Miranda, "Treating depressed older adults in primary care: narrowing the gap between efficacy and effectiveness", The Milbank Quarterly, 1999, pp. 225-256. 
[11] S. A. Kinner, N. Lennox, G. M. Williams, M. Carroll, B. Quinn, F. M. Boyle and R. Alati, "Randomised controlled trial of a service brokerage intervention for ex-prisoners in Australia", Contemporary Clinical Trials, 2013, pp. 198-206.

[12] S. A. Spohr, F. S. Taxman and S. T. Walters, "The relationship between electronic goal reminders and subsequent drug use and treatment initiation in a criminal justice setting", Addictive Behaviors, 2015, pp. 51-56.

[13] S. T. Walters, S. J. Ondersma, K. S. Ingersoll, M. Rodriguez, J. Lerch, M. E. Rossheim and F. S. Taxman, "MAPIT: Development of a web-based intervention targeting substance abuse treatment in the criminal justice system", Journal of Substance Abuse Treatment, 2014, pp. 60-65.

[14] Pew Research Center, "Mobile Fact Sheet", in 2021. [Online]. https://www.pewresearch.org/internet/factsheet/mobile/. [Accessed 06/12, 2021]

[15] B. J. U. Castonguay, A. E. Cressman, I. Kuo, R. Patrick, C. Trezza, A. Cates, H. Olsen, J. Peterson, A. Kurth, L. B. Bazerman and C. G. Beckwith, "The Implementation of a Text Messaging Intervention to Improve HIV Continuum of Care Outcomes Among Persons Recently Released From Correctional Facilities: Randomized Controlled Trial", Jmir Mhealth and Uhealth, 2020, pp.

[16] F. S. Taxman, "Community supervision in the post mass incarceration era", Fed. Probation, 2015, pp. 41.

[17] M. Tolou-Shams, J. Yonek, K. Galbraith and E. Bath, "Text Messaging to Enhance Behavioral Health Treatment Engagement Among Justice-Involved Youth: Qualitative and User Testing Study", Jmir Mhealth and Uhealth, 2019, pp.

[18] J. M. R. Gonzalez, M. S. Businelle, D. Kendzor, M. Staton, C. S. North and M. Swartz, "Using mHealth to Increase Treatment Utilization Among Recently Incarcerated Homeless Adults (Link2Care): Protocol for a Randomized Controlled Trial", Jmir Research Protocols, 2018, pp.

[19] N. F. Sugie and M. C. Lens, "Daytime Locations in Spatial Mismatch: Job Accessibility and Employment at Reentry From Prison", Demography, 2017, pp. 775-800.

[20] I. F. Ogbonnaya-Ogburu, K. Toyama and T. R. Dillahunt, "Towards an Effective Digital Literacy Intervention to Assist Returning Citizens with Job Search", in Proceedings of the 2019 CHI Conference on Human Factors in Computing Systems, Association for Computing Machinery, Glasgow, Scotland Uk, 2019 of Conference, pp. Paper 85.

[21] C. Abras, D. Maloney-Krichmar and J. Preece, "Usercentered design", Bainbridge, W. Encyclopedia of Human-Computer Interaction. Thousand Oaks: Sage Publications, 2004, pp. 445-456.

[22] C. Bickerton, A. Gourevitch, L. Jones and P. Ramsay, "Mass Incarceration follow up", in The Current Moment 2012. [Online]. Available: https://thecurrentmoment.wordpress.com/2012/06/01/m ass-incarceration-follow-up/. [Accessed 3/5, 2021]

[23] J. Bronson and M. Berzofsky, "Indicators Of Mental Health Problems Reported By Prisoners And Jail Inmates, 2011-2012”, in Bureau of Justice Statistics,
2017.

[Online].

Available: https://www.bjs.gov/index.cfm?ty=pbdetail\&iid=5946. [Accessed 3/5, 2021]

[24] J. Bronson, J. Stroop, S. Zimmer and M. Berzofsky, "Drug Use, Dependence, And Abuse Among State Prisoners And Jail Inmates, 2007-2009”, in Bureau Of Justice Statistics, 2017. [Online]. Available: https: $/ /$ www.bjs.gov/index.cfm?ty=pbdetail\&iid $=5966$. [Accessed 2/23, 2021]

[25] M. Sarchiapone, N. Jovanović, A. Roy, A. Podlesek, V. Carli, M. Amore, M. Mancini and A. Marušič, "Relations of psychological characteristics to suicide behaviour: Results from a large sample of male prisoners", Personality and Individual Differences, 2009, pp. 250255.

[26] N. E. Fearn, M. G. Vaughn, E. J. Nelson, C. P. SalasWright, M. DeLisi and Z. Qian, "Trends and correlates of substance use disorders among probationers and parolees in the United States 2002-2014", Drug and alcohol dependence, 2016, pp. 128-139.

[27] J. Tsai and X. Gu, "Utilization of addiction treatment among U.S. adults with history of incarceration and substance use disorders", Addict Sci Clin Pract, 2019, pp. 9.

[28] J. Baillargeon, I. A. Binswanger, J. V. Penn, B. A. Williams and O. J. Murray, "Psychiatric disorders and repeat incarcerations: the revolving prison door", American Journal of Psychiatry, 2009, pp. 103-109.

[29] J. Bronson, L. M. Maruschak and M. Berzofsky, "Disabilities among prison and jail inmates, 2011-12", US Department of Justice Bureau of Justice Statistics, 2015, pp.

[30] D. E. McNiel, R. L. Binder and J. C. Robinson, "Incarceration associated with homelessness, mental disorder, and co-occurring substance abuse", Psychiatric Services, 2005, pp. 840-846.

[31] A. B. Wilson, J. Draine, T. Hadley, S. Metraux and A. Evans, "Examining the impact of mental illness and substance use on recidivism in a county jail", International journal of law and psychiatry, 2011, pp. 264-268.

[32] I. A. Binswanger, M. F. Stern, R. A. Deyo, P. J. Heagerty, A. Cheadle, J. G. Elmore and T. D. Koepsell, "Release from prison - a high risk of death for former inmates", New England Journal of Medicine, 2007, pp. 157-165.

[33] S. I. Ranapurwala, M. E. Shanahan, A. A. Alexandridis, S. K. Proescholdbell, R. B. Naumann, D. Edwards, Jr. and S. W. Marshall, "Opioid Overdose Mortality Among Former North Carolina Inmates: 2000-2015", American journal of public health, 2018, pp. 1207-1213.

[34] R. K. Chandler, B. W. Fletcher and N. D. Volkow, "Treating drug abuse and addiction in the criminal justice system: improving public health and safety", Jama, 2009, pp. 183-90.

[35] "Principles of Community-based Behavioral Health Services for Justice-involved Individuals: A Researchbased Guide", in Substance Abuse and Menta Health Services Administration (SAMHSA), 2019. [Online]. Available: https://store.samhsa.gov/product/Principlesof-Community-based-Behavioral-Health-Services-for- 
Justice-involved-Individuals-A-Research-basedGuide/SMA19-5097. [Accessed 06/13, 2021]

[36] M. Arceneaux, "Why Are The Three Largest Mental Health Care Providers Jails?", in Newsone, 2013. [Online]. Available: https://newsone.com/2744141/prisons-mental-healthproviders/. [Accessed 4/3, 2021]

[37] S. J. Bahr, A. L. Masters and B. M. Taylor, "What works in substance abuse treatment programs for offenders?", The Prison Journal, 2012, pp. 155-174.

[38] J. Bronson, J. Stroop, S. Zimmer and M. Berzofsky, "Drug use, dependence, and abuse among state prisoners and jail inmates, 2007-2009”, Washington, DC: United States Department of Justice, Office of Juvenile Justice and Delinquency Prevention, 2017, pp.

[39] K. Mallik-Kane and C. A. Visher, Health and prisoner reentry: How physical, mental, and substance abuse conditions shape the process of reintegration, Urban Institute Justice Policy Center Washington, DC, 2008.

[40] F. S. Taxman, A. Pattavina and M. Caudy, "Justice reinvestment in the United States: An empirical assessment of the potential impact of increased correctional programming on recidivism", Victims \& Offenders, 2014, pp. 50-75.

[41] F. S. Taxman, M. L. Perdoni and M. Caudy, "The plight of providing appropriate substance abuse treatment services to offenders: Modeling the gaps in service delivery", Victims \& Offenders, 2013, pp. 70-93.

[42] American Psychological Association, "From prisons to communities: Confronting re-entry challenges and social inequality", in 2018. [Online]. Available: https://www.apa.org/pi/ses/resources/indicator/2018/03/ prisons-to-communities. [Accessed 4/1, 2021]

[43] National Health Care for the Homeless Council, "Homelessness \& Health: What's the Connection?", in 2019. [Online]. Available: https://nhchc.org/wpcontent/uploads/2019/08/homelessness-and-health.pdf. [Accessed 2021,

[44] E. L. Grommon, Prisoner reentry programs: Penetrating the black box for better theory and practice, LFB Scholarly Pub., 2013.

[45] K. Burrowes, "Can Housing Interventions Reduce Incarceration and Recidivism?", in Housing Matters, 2019. [Online]. Available: https://housingmatters.urban.org/articles/can-housinginterventions-reduce-incarceration-and-recidivism. [Accessed 3/27, 2021]
[46] N. M. Morani, N. Wikoff, D. M. Linhorst and S. Bratton, "A description of the self-identified needs, service expenditures, and social outcomes of participants of a prisoner-reentry program", The Prison Journal, 2011, pp. 347-365.

[47] A. B. Laudet, V. Stanick and B. Sands, "What could the program have done differently? A qualitative examination of reasons for leaving outpatient treatment", Journal of substance abuse treatment, 2009, pp. 182-190.

[48] S. A. Spohr, S. T. Walters and F. Taxman, "The relationship between electronic goal reminders and subsequent drug use and treatment initiation in a criminal justice setting", Addictive Behaviors, 2015, pp. 51-56.

[49] D. Chrimes, N. R. Kitos, A. Kushniruk and D. M. Mann, "Usability testing of Avoiding Diabetes Thru Action Plan Targeting (ADAPT) decision support for integrating care-based counseling of pre-diabetes in an electronic health record", Int J Med Inform, 2014, pp. 636-47.

[50] B. Wagner, A. B. Horn and A. Maercker, "Internet-based versus face-to-face cognitive-behavioral intervention for depression: a randomized controlled non-inferiority trial", Journal of affective disorders, 2014, pp. 113-121.

[51] S. C. King, K. A. Richner, A. P. Tuliao, J. L. Kennedy and D. E. McChargue, "A comparison between telehealth and face-to-face delivery of a brief alcohol intervention for college students", Subst Abus, 2020, pp. 501-509.

[52] L. A. Marsch, K. M. Carroll and B. D. Kiluk, "Technology-based interventions for the treatment and recovery management of substance use disorders: a JSAT special issue", Journal of substance abuse treatment, 2014, pp. 1-4.

[53] W. E. K. Lehman, J. Pankow, G. A. Rowan, J. Gray, T. R. Blue, R. Muiruri and K. Knight, "StaySafe: A selfadministered android tablet application for helping individuals on probation make better decisions pertaining to health risk behaviors", Contemporary Clinical Trials Communications, 2018, pp. 86-93.

[54] J. Brooke, "SUS: a retrospective", Journal of usability studies, 2013, pp. 29-40.

[55] E. L. Deci and R. M. Ryan, "Self-determination theory", 2012, pp.

[56] L. Faulkner, "Beyond the five-user assumption: Benefits of increased sample sizes in usability testing", Behavior Research Methods, Instruments, \& Computers, 2003, pp. 379-383. 